\title{
Examination of Infrared Tomato Drying
}

\author{
Zs. VÁRKONYI ${ }^{1}$, V. SEBESI ${ }^{2}$, M. ÖRVÖS ${ }^{3}$ \\ ${ }^{1}$ Budapest University of Technology and Economics, Faculty of Mechanical Engineering, Department of Building \\ Service Engineering and Process Engineering, zsombor.varkonyi@gmail.com \\ 2Budapest University of Technology and Economics, Faculty of Mechanical Engineering, Department of Building \\ Service Engineering and Process Engineering, sebesi.viki@gmail.com \\ ${ }^{3}$ Budapest University of Technology and Economics, Faculty of Mechanical Engineering, Department of Building \\ Service Engineering and Process Engineering, orvos@mail.bme.hu
}

Abstract. Tomato is a highly perishable food, thus preservation is required to meet the continuous market demand compared to the unbalanced yearly production peak. The aim of this paper is to show a detailed insight into infraradiation based drying process. Furthermore, to widen the possible usage range of a well-known approximation method for describing the temporal moisture variation in function of process parameters. The mass decrease of tomato slices was examined, in function of specific radiation power, heat source and sample distance, in forced and natural airflow. Based on the results dimensionless moisture ratio change over time was fitted as an exponential function, which may be used in industrial drying process optimization.

\section{Introduction}

Many experiments were conducted regarding the process parameters of infrared drying. H. Kocabiyik [1] found that while at convective drying the increased velocity of drying gas results in better heat transfer, thus quicker drying, then it is completely the opposite with radiation driven process. From the three checked options $(1 ; 1,5 ; 2 \mathrm{~m} / \mathrm{s})$ the higher speed meant slower drying, due to the cooling effect of ambient air. This is corresponding with the founding of G.P. Sharma [2], who IR dried onion slices on $1 ; 1,25$ and 1,5 m/s. Furthermore, the results shown that this correlation is true at much different radiation source power and different type of vegetables as well.

Another important factor for the process is the quality of the resulted product after the thermal treatment. In the paper of Kocabiyik the drying time, specific energy consumption, shrinking, color, vitamin $\mathrm{C}$ and lycopene content was represented at 1830, 2385, 2640, 2880, $3165 \mathrm{~W} / \mathrm{m} 2$ radiation power and different ambient air speeds. Shrinkage (volume ratio of processed and fresh tomato) is 0,139-0,203, mostly independent from drying parameters. The lowest specific energy consumption happened at $1 \mathrm{~m} / \mathrm{s}$ and $2640 \mathrm{~W} / \mathrm{m} 2$. Vitamin $\mathrm{C}$ and beta-carotene content decreased at all time, but lycopene significantly increased at many occasions, which is an important advantage compared to conventional hot-air drying.

Furthermore G. P. Sharma found that the required time is inversely proportional to the surface or core temperature of sample during the procedure. However, it is also important to mention that over a certain temperature (approximately $60^{\circ} \mathrm{C}$ ) the decomposition of lycopene and ascorbic-acid intensifies 
exponentially. Moreover, at industrial sized processes the generation of high temperature is maybe more energy demanding, than the profit, which is won by achieving shorter process time.

Above these the moisture content of the ambient air is an essential parameter to set the drying process well. The sorption isotherm - which determines the lowest achievable moisture content - is well described in the literature. For example, the research of Ginzburg and Akanbi [3] shows achievable dry matter based water content versus relative moisture of air at different process parameters. While according to T. M. Afzal [4] it can be stated that the humidity of air has almost no effect on the speed of infrared drying of potato slices, but only the final water content of product.

Based on the wide range of available literature it can be stated that infrared drying of tomato and other vegetables is well examined and the effect of changing various parameters are approximately known based on the results. The aim of this paper is to further broaden the knowledge by giving the parameters of a commonly used temporal mass decrease expression in function of process parameters.

\section{Mathematical description}

Based on the theoretical description of radiation, the transferred heat is proportional to the surface of source, emission factor of both elements, geometric relations and the difference of the absolute temperature of the two objects on the power of four. The total radiated heat glows on a theoretical area and only a proportional part reaches the tomato itself, which effect should also be taken into account as in Eq. (1). The received heat $Q_{\text {received }}$ is used for increasing the temperature of tomato Qtemp.raise (which duty is negligible due to the low heat capacity compared to the latent heat), evaporating water $Q_{\text {evaporation }}$ (the speed of which is rather defined by diffusion) and heat losses Q $_{\text {losses. }}$. The overall heat balance can be described as shown in Eq. (2). Assuming that the temperature raising part is negligibly short and the losses are constant, they can be moved to the right side of the equation, while the evaporation of water is interpretable as temporal mass decrease times latent heat as in Eq. (3). Supposing a generally accepted constant dry matter during the process the mass change can be expressed as evaporated water, which is a moisture content derivative Eq. (4).

$$
\begin{gathered}
Q_{\text {received }}=A_{\text {source }} \cdot \sigma \varepsilon_{1} \varepsilon_{2} \varphi \cdot\left(T_{\text {source }}^{4}-T_{\text {tomato }}^{4}\right) \frac{A_{\text {tomato }}}{A_{\text {surface }}} \\
Q_{\text {received }}=Q_{\text {evaporation }}+Q_{\text {temp.raise }}+Q_{\text {losses }} \\
Q_{\text {evaporation }}=\frac{d m_{\text {tomato }}}{d t} \cdot r_{p} \cong A_{\text {source }} \cdot \sigma \varepsilon_{1} \varepsilon_{2} \varphi \cdot\left(T_{\text {source }}^{4}-T_{\text {tomato }}^{4}\right) \frac{A_{\text {tomato }}}{A_{\text {surface }}}-Q_{\text {losses }} \\
\frac{d m_{\text {tomato }}}{d t}=\frac{d m_{H 2 O}}{d t}=\frac{d\left(X \cdot m_{\text {dry matter })}\right.}{d t}=m_{\text {dry matter }} \cdot \frac{d X}{d t}
\end{gathered}
$$

It is a common habit to describe a process with dimensionless numbers where possible, to avoid conversion problems. In this case the baseline reference of a dimensionless expression is the dry matter based water content $\mathrm{X}$. The widely used moisture ratio MR is the moisture at given moment $\mathrm{X}(\mathrm{t})$, divided by the initial value $\mathrm{X}_{0}$ (supposing the equilibrium $\mathrm{X}_{\mathrm{e}}$ is significantly smaller than the before mentioned two other). 


$$
M R(t)=\frac{X(t)-X_{e}}{X_{0}-X_{e}} \approx \frac{X(t)}{X_{0}}
$$

In case of knowing the moisture ratio in function of time, the $\mathrm{X}(\mathrm{t})$ can be obtained, from what the derivative, thus the heat requirement is given as well. The $M R=f(t)$ function was given by many authors according to the collection of Ali Mohammadi [5]. Solving the first order differential equation (3) shows that the change of weight in function of time be estimated with an exponential expression, thus most of the approximate functions are in this form.

$$
M R(t)=a \cdot e^{-b \cdot t}
$$

The expression (6) of Henderson-Pabis is broadly used for radiational [5] and convectional [6] drying processes, as it is quite simple, usually fits the experimental results well and the theoretical background can be easily seen.

In Eq. (6) the MR is given in function of time, in an exponential form, where a is a dimensionless number to describe the initial or residual water content while factor $b[1 / \mathrm{s}]$ defines the time dependent change.

\section{Measurement Method}

The experiments [7] were conducted with approximate 60-70g weight, round, slightly hard, evenly red colour common hungarian tomato type (Lycopersicon lycopersicum). According the literature $8 \mathrm{~mm}$ thick, $60 \pm 5 \mathrm{~mm}$ diameter, round slices were dried to avoid quick burning, but also to achieve relative quick drying procedure.

To conclude the experiments a special measurement method and a unique laboratory station was built. A 250W Tungsram IR bulb (1) was placed on a stand. Under it the tomato sit on a welded grid (3) - to ensure airflow under the product too -, while it is placed on a Sartorius Bp 3100 balance (2). The initial weight was measured on an EMB 2000-2 high precision balance as verification. The surface temperature was measured by an infra-thermometer (5), which was beforehand calibrated with a contact thermometer. The data recorder (7)- connected to a PC (6) - saved the physical properties of ambient air (8), the surface temperature, core temperature and the mass every half minute during the experiment. The tomato slice (4) - situated from a given height distance under the heat source - was blown from the side by a fan (9) with given speed ambient air. To minimize possible error sources nearby windows were blinded. The exact electric consumption of light bulb was measured at the electric socket (11).

The instrumentation was assembled in a way that all required parameters can be measured to obtain as exact results as possible. Ambient air temperature, absolute and relative moisture, inner- and surface temperature, as well as weight of tomato slice were saved with the above mentioned frequency. Speed of air was determined using an impeller type wind speed meter, while the power of the lightbulb was measured with a household socket electric consumption meter. 


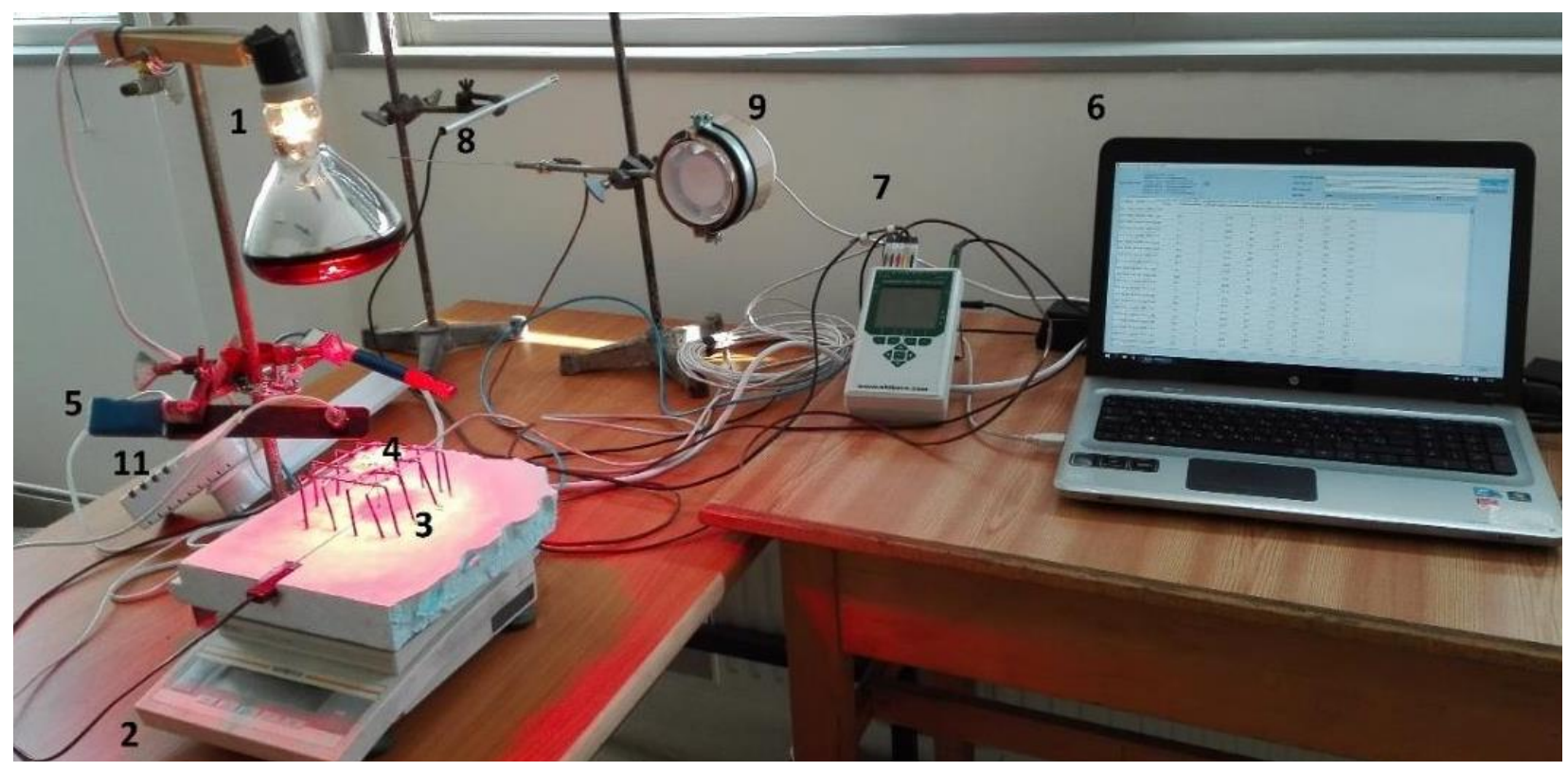

Figure 1. Measurement setup

Some other data were checked time to time during the whole experiment. The edge of the radiated surface was determined with an AMB-170 light meter, based on the significantly changing lux value, besides optical checking. The shape of the tomato slice was assumed an ellipse. The length of the two axis were measured before and after the drying process and from the calculated surfaces shrinkage was given. Emissivity factor was measured with the calibration of a manual infra thermometer to a contact thermometer.

Initial moisture content was also determined using a desiccator for all tomatoes. The weight of the probe was measured before and after being placed into a drying chamber at $105^{\circ} \mathrm{C}$ for 24 hour. The calculated average $95.3 \%$ water content matches well the available literature data.

\section{Experimental results}

The experiments were concluded with several different parameters. The probe to radiation source distance was set to 100,125, 150, 175, 200 and $250 \mathrm{~mm}$. From the height of the gap, the angle of the wall of light bulb the radiated surface can be calculated, which together with the measured $231 \pm 1 \mathrm{~W}$ power determines the surface specific heat duty as well: $0.01808 ; 0.00802 ; 0.00451 ; 0.00295 \mathrm{~W} / \mathrm{mm}^{2}$ accordingly. The last value of $2950 \mathrm{~W} / \mathrm{m}^{2}$ fits well into the range of different intensities used by Kocabiyik [1]. The other values are slightly bigger, because the distance was minimized in this experiment to get results also from a less widely studied area.

Besides this, at half of the cases the forced ambient air speed was $1 \mathrm{~m} / \mathrm{s}$ from the side, while at others it was $0 \mathrm{~m} / \mathrm{s}$, only natural flow happened, to see if this parameter has any significant advantage compared to the known heat loss detriment. 


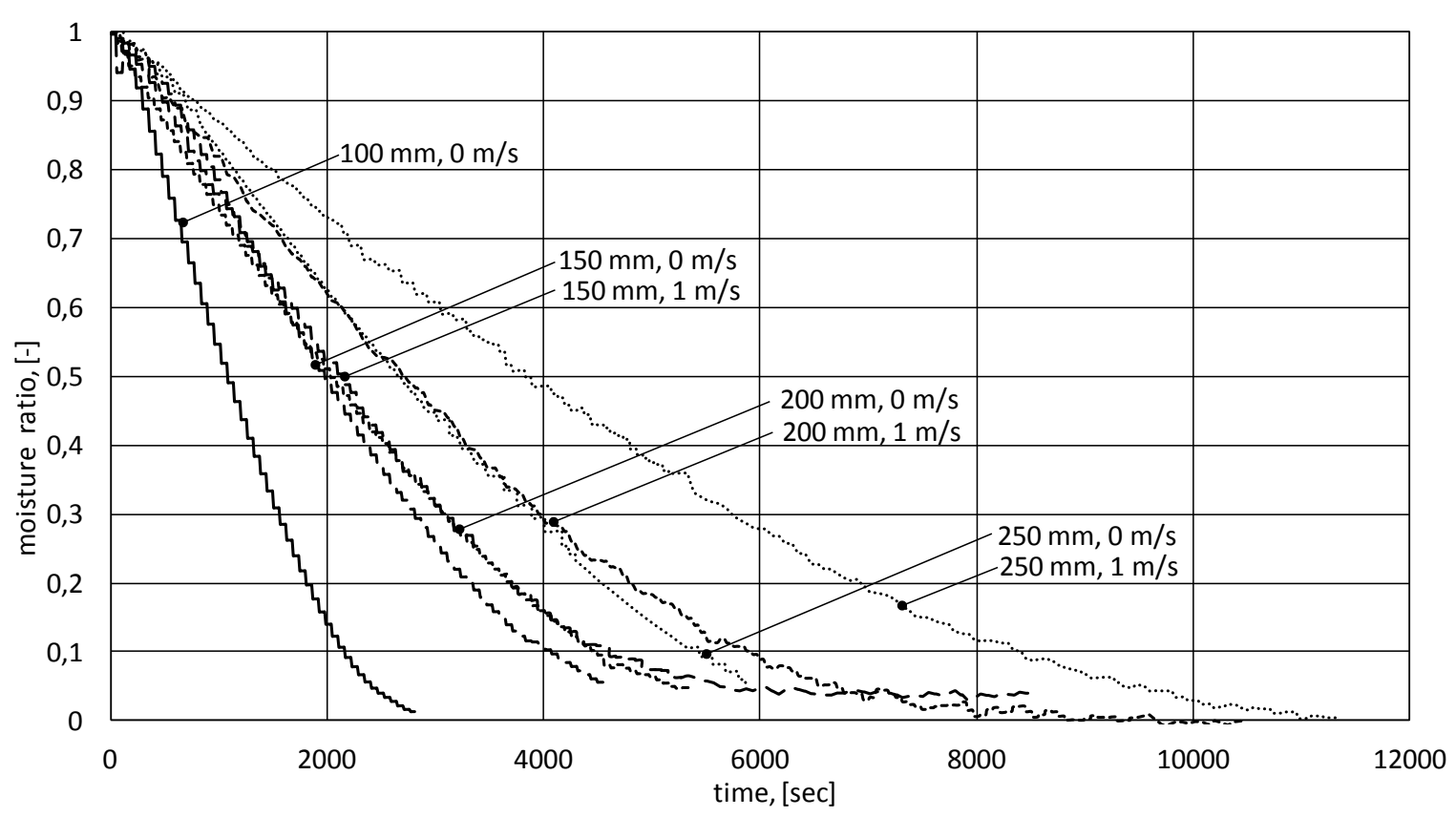

Figure 2. Moisture ratio decrease at different drying parameters

Figure 2 shows how the moisture ratio changed in function of time during the different process options. The same type dotted curves mean same distance and the legend helps to distinguish between blown and non-blown cases 1 . First it can be seen that - when every process parameter was similar, except air speed - the curves are steeper in the case of zero gas flow. This means it takes less time to reach similar moisture content, thus the drying process is quicker. At convectional drying the higher velocity means higher Reynolds number, so better, quicker heat transfer. This turns into a disadvantage at infrared drying, because here the conductive heat transfer is minimized and the generally cooler ambient air only causes heat loss. Therefore, from point of process speed using forced flow ambient air is a disadvantage, even $1 \mathrm{~m} / \mathrm{s}$ almost doubled the process time, matching the literature data $[1,2]$.

On the other hand, the quicker, more energy efficient, but not so gentle procedure influenced the product quality a lot. For example, the ratio of final versus initial slice area was about 0.58 when there was no air flow and about 0.65 in the other case, additionally independent from other process parameters. The bigger shrinkage generally means bigger deformation, thus more amorphous, less appealing end product.

Furthermore, the diagram shows that - when every process parameter was similar, except tomato to bulb gap - the curves are steeper in the case of smaller radiation source to probe gap height. This happens, because the specific power - as well the heat inlet to tomato - is higher, which naturally results in faster drying process. However putting closer the heat source affects the temperature in a disadvantageous way. $100 \mathrm{~mm}$ difference in the distance meant approximately $10^{\circ} \mathrm{C}$ variation in the core and more than $15^{\circ} \mathrm{C}$ on the surface temperature. For example, with the theoretically quickest process parameters $(100 \mathrm{~mm}$ between the two objects, no artificial air flow) the surface reached average $80^{\circ} \mathrm{C}$ during the constant drying rate phase and more than $120^{\circ} \mathrm{C}$ later, which of course ended up in burnt tomato. 


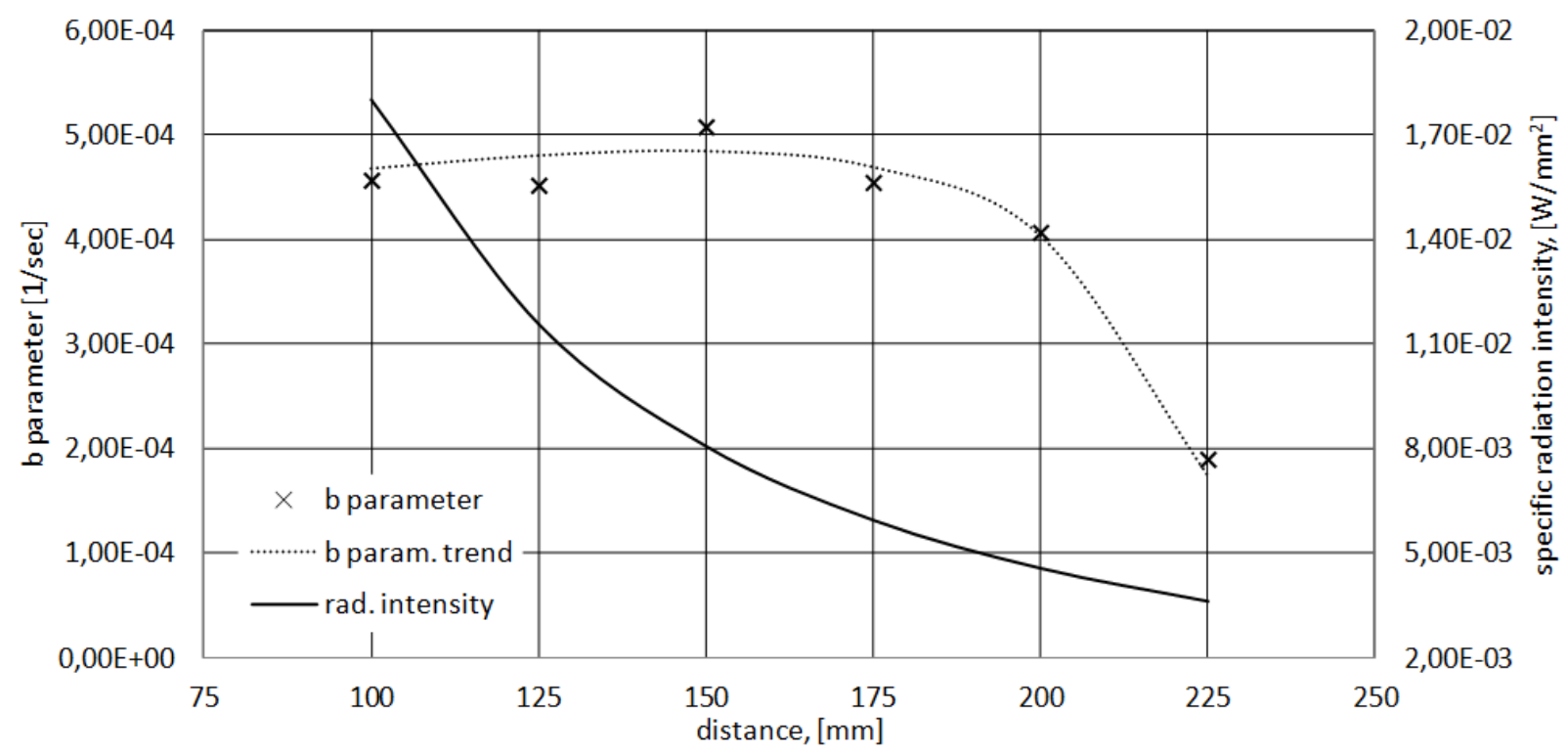

Figure 3. Constant $b$ of H-P model

Due to the fact that, the tomatoes were able to dry to various rates at different drying parameters, results were evaluated and used until $42 \%$ wt water content $(M R=0.05)$ for comparison and calculation. The expression of Henderson-Pabis model Eq. (4) is quite simple for this case. At the initial time moment $M R=1$, thus the value of " $a$ " parameter is 1 . Parameter " $b$ " can be obtained from the rearranged equation. After calculating the parameter, the theoretical and experimental results were compared and the results are satisfactory, error square $\mathrm{R}^{2}$ is bigger than 0.8 at any cases.

The constant $\mathrm{b}$ versus tomato to light bulb distance values for the non-blown case can be found in Figure 3, taking into consideration all experimental parameters described in measurement method paragraph as conditions. From the correlation it can be realized that the connection has a decreasing characteristic. This was expected, as the $\varphi$ parameter describing geometric relation in Eq. (3) - thus the specific radiation duty - is exponentially decreasing with the increased distance.

\section{Conclusion}

Within the frames of this project an experimental method and measuring station was composed to examine infrared food drying process. The light bulb to tomato slice distance and natural versus low speed forced airflow were the two main parameters which were reviewed. Based on the experimental results a widely applied mathematical expression was fitted to describe the moisture ratio in function of time. The $b$ parameter of the Henderson-Pabis model was determined for $100-250 \mathrm{~mm}$ radiation source to probe distance (3000-18000 W/ $\mathrm{m}^{2}$ specific heat duty), for $0 \mathrm{~m} / \mathrm{s}$ air flow speed, for the 1 to 0.05 moisture ratio range. Based on the experimental dataset a function was established to determine the $b[1 / s]$ parameter in function of tomato to bulb distance $h[\mathrm{~mm}]$, shown in Eq. (7). So the MR(t), $X(t), m(t), d m / d t$, thus finally the required heat duty $Q(t)$ (Eqs. 3-6) can be estimated.

$$
b=\frac{0,0004011024--0,000001721713 \cdot h}{1-0,005908795 \cdot h+0,000008064992 \cdot h^{2}}
$$


The drying process time until the $8 \mathrm{~mm}$ thick $60 \mathrm{~mm}$ diameter tomato slices reach $\mathrm{MR}=0.05$ value with $231 \mathrm{~W}$ infrared light bulb, in function of radiation source to product distance, in case of $1 \mathrm{~m} / \mathrm{s}$ or no forced ambient air flow can be also estimated applying the given parameters in the Handerson-Pabis model.

Generally, it can be stated that the setting of drying parameters significantly affects the required time of process along with the quality of the end product.

During the evaluation the heat loss from the surface of the tomato and the heat required for increasing the temperature of the sample until the constant drying speed phase was neglected. The model constant $\mathrm{b}$ of H-P model contains all the collateral heat losses or increments, which shall be taken into consideration during the usage of Eq. (7).

Using radiation as primary heat source combined with low, but higher than ambient temperature air to ensure ideal conditions for good quality along with efficient process - could be an other possible examination topic. In the future we would like to continue the experiments and based on the results examine, select, and determine parameters of other temporal moisture variation expressions, with lower error and wider possible usage range.

\section{Acknowledgement}

The research reported in this paper was supported by the Higher Education Excellence Program of the Ministry of Human Capacities in the frame of Water science \& Disaster Prevention research area of Budapest University of Technology and Economics (BME FIKP-VÍZ).

The devices used in this research were supported by the National Talent Program - National Young Talent Scholarship of the Ministry of Human Capacities (NTP NFTÖ 2018).

The authors would like to thank Dr. Tibor Poós for all his help granted during the measurements.

\section{References}

[1] H. Kocabiyik et al. (2014) The effects of middle infrared radiation intensity on the quality of dried tomato products. Int. J. Food Sci. Technol., 49, pp. 703-710.

[2] G.P. Sharma et al. (2005) Thin-layer infrared radiation drying of onion slices. J. Food Eng. 67 (3) pp. 361-366.

[3] C.T. Akanbi et al. (2006) Drying characteristics and sorption isotherm of tomato slices. J. Food Eng. 73 (2) pp. 157-163.

[4] T. M. Afzal - T. Abe (1999) Some fundamental attributes of far infrared radiation drying of potato. Dry. Technol. 17, pp. 138-155.

[5] Moisture Content Modeling of Sliced Kiwifruit During Drying. [Online].: http://scialert.net/fulltext/?doi=pjn.2009.78.82. [06-nov-2017].

[6] Gy. Schneider et al. (2014) Energy consumption of tomato drying. Proceedings of ISCAME. pp. 111-116.

[7] V. Sebesi et al. (2017) Infrasugaras paradicsomszárítás. Budapest University of Technology and Economics, BSc thesis, 2017. 